\title{
Vibration isolation performance of an elevator motor using Nitrile-Butadiene Rubber /Multi- Walled Carbon Nanotube composite machine mounts
}

\author{
Konstantinos Tsongas ${ }^{1, *}$, Gabriel Mansour ${ }^{1}$ \\ ${ }^{1}$ Laboratory for Machine Tools and Manufacturing Engineering, Department of Mechanical \\ Engineering, Aristotle University of Thessaloniki, University Campus, 54124, Thessaloniki, Greece.
}

\begin{abstract}
The objective of this paper is to evaluate the vibration isolation performance of an elevator motor mounted on elastomeric nanocomposite mounts. A series of conventional acrylonitrile-butadiene rubber (NBR) mounts have been reinforced with $20 \mathrm{wt} \%$ concentration of multi-walled carbon nanotubes (MWCNTs). The vibration isolation capacity of the machine mounts was calculated through the transmissibility of an elevator motor test system. A Finite Element Model (FEM) was introduced and a harmonic analysis based on the ANSYS code has been utilized to investigate the modal behavior of the nanocomposite machine mount/elevator motor system and extract a representative model of the vibrational behavior. The cyclic compression results have revealed that the stiffness and damping capacity of the conventional elastomers can be modified by adjusting the proportion of MWCNTs. Elastomers' vibration isolation performance of the motor was ameliorated with the inclusion of MWCNTs, signifying that the enhancement of the elastomers with MWCNTs was rather effective. The vibration level of the elevator motor was decreased to $90 \%$ by incorporating the optimal concentration of MWCNTs in NBR mounts.
\end{abstract}

\section{Introduction}

Conventional elevators move vertically by the traction machine, which suspends the car and the counter weight by the rope. Elevators in residential buildings present the potential for intrusive noise and vibration to the dwelling units. The adverse noise and vibration can result from the elevator equipment located in the machine rooms or the shafts. The impacts can be significant issues related to the sound quality, sleeping conditions, and enjoyment within residences. Resilient mounting is widely used in vibration and noise control to isolate a vibratory source from a receiving structure or from a structural component with a significant radiating area. In order to design an effective isolator or to predict the level of isolation, an accurate measurement of the dynamic behavior, i.e. dynamic complex stiffness and damping, is necessary. The concept of complex stiffness with viscous and hysteresis damping has been

\footnotetext{
*Corresponding author: ktsongas@auth.gr
} 
exquisitely explained for a single degree of freedom (SDOF) system [1]. Numerous test methods have been developed in order to characterize the complex modulus properties of elastomeric machine mounts $[2,3]$. In the past, the damping capacity of conventional engineering materials does not always provide sufficient energy dissipation to limit resonant or near-resonant amplitudes of vibration [4]. High damping capacity is an important factor for various industrial applications; therefore, it is necessary to develop structural components with a high level of structural damping. It would therefore be of interest to investigate new materials that simultaneously exhibit high damping capacity with high stiffness and low density which includes rubber matrix nanocomposites and porous metals.

High loss factor polymers have been used in the past for modern damping applications [5-7]. Nowadays there are various other advanced materials that could be utilized in vibration isolation applications. Researchers have concentrated their efforts towards the development of polymers in which nanomaterials are embedded in polymer substrates and much promising results have been established primarily for improvement of the mechanical properties [8-12]. The interest in porous metals arises from the interesting combination of characteristics and the easy adjustment of their properties to meet the desired needs by tailoring porosity characteristics.

In order to assess the vibration isolation performance of such high stiffness and high damping materials, a modal testing method proposed by Mansour et al. [13-18] derives modal parameters from experimental transfer functions by a curve-fitting technique, in order to determine preliminary modal parameters, from which analytical expressions for the TFs are obtained.

The vibration isolation behavior of an elevator motor machine isolated by MWCNTs/NBR elastomers is the subject of the current investigation. In order to obtain the modal properties of the elevator motor machine with nanocomposite isolators, a finite element model was developed. Loading-unloading (cyclic) compression tests were performed in order to determine the stiffness and damping capacity, as input for the finite element model. The vibration isolation performance of the elevator motor was characterized by analyzing the resonant frequencies, the damping and the transmissibility of the nanomodified elastomeric system. This procedure was repeated for the different nanofiller concentrations in the elastomeric matrix. The nanocomposite elastomers were considered optimal for the vibration isolation of the elevator motor machine.

\section{EXPERIMENTAL DETAILS}

\subsection{Cyclic compression tests of NBR/MWCNTs nanocomposite machine mounts}

At least five nanocomposite specimens were prepared with 20wt $\%$ MWCNTs/NBR, as well as $0 \mathrm{wt} \% \mathrm{MWCNTs} / \mathrm{NBR}$ which served as a control sample. Subsequently, MWCNTs/rubber elements were bonded between a steel pedestal and steel cap. The cap provides protection for the rubber element from diesel, oil, ozone, UV rays that are harmful to rubber. The final dimensions of the NBR/MWCNTs mounts were $60 \mathrm{~mm}$ diameter and $27 \mathrm{~mm}$ height.

Steady state, strain rate-controlled cyclic compression tests at ambient temperature were performed with constant strain rates in loading and unloading. The measurement was carried out using a material testing system (Testometric, UK equipped with a $50 \mathrm{kN}$ load cell) at a frequency of $0.01 \mathrm{~Hz}$ and up to a $10 \mathrm{kN}$ load. The loss of energy in the loading-unloading cycle for each material was calculated from the hysteresis loop. The stress-strain behavior during loading and unloading was obtained and each rubber demonstrated a hysteresis behavior. The loading and unloading speeds were set constantly at $5 \mathrm{~mm} / \mathrm{min}$. 


\section{MECHANICAL AND HYSTERETIC BEHAVIOR OF THE MWCNTS NANOCOMPOSITE ELASSTOMERIC MOUNTS}

\subsection{Mechanical and damping properties of the NBR/MWCNTs nanocomposite mounts}

Under alternating stress, hysteresis occurs when the rate of deformation is less than the rate of stress variation. In this case, since the absorbed and released energies are not balanced in each cycle, the stretching and recoil curve form a closed loop, which is known as a hysteresis loop as shown in Figure 1. The area within the loop represents the energy loss. For elastomeric materials, a larger hysteresis loop means higher damping, which more effectively reduces vibration [19]. The damping constants may be derived from the area surrounded by hysteresis loops. Based on the theory of free vibration, the vibration-isolating capacity of materials can be evaluated from the damping constant and the hysteresis damping characteristics [20].

The specific damping capacity (SDC) is given by:

$$
\begin{gathered}
S D C=\frac{\Delta W}{W} \times 100 \%=\left(\oint \sigma d \varepsilon / \int_{\omega t=0}^{\pi / 2} \sigma d \varepsilon\right) \times 100 \% \\
=\left(\pi \cdot \sigma_{0} \cdot \varepsilon_{0} \cdot \sin \delta\right) /\left(\frac{1}{2} \cdot \sigma_{0} \cdot \varepsilon_{0}\right. \\
\cdot \cos \delta)=2 \pi \tan \delta
\end{gathered}
$$

where $\sigma$ and $\varepsilon$ are the stress and strain, $\delta$ is the phase angle between them, $\Delta W$ is the energy dissipated in any one cycle and $W$ is the maximum energy associated with that cycle [21]. The specific damping capacity can be related with the loss factor [22] by:

$$
n=\tan \delta=\frac{\Delta W}{2 \pi W}
$$

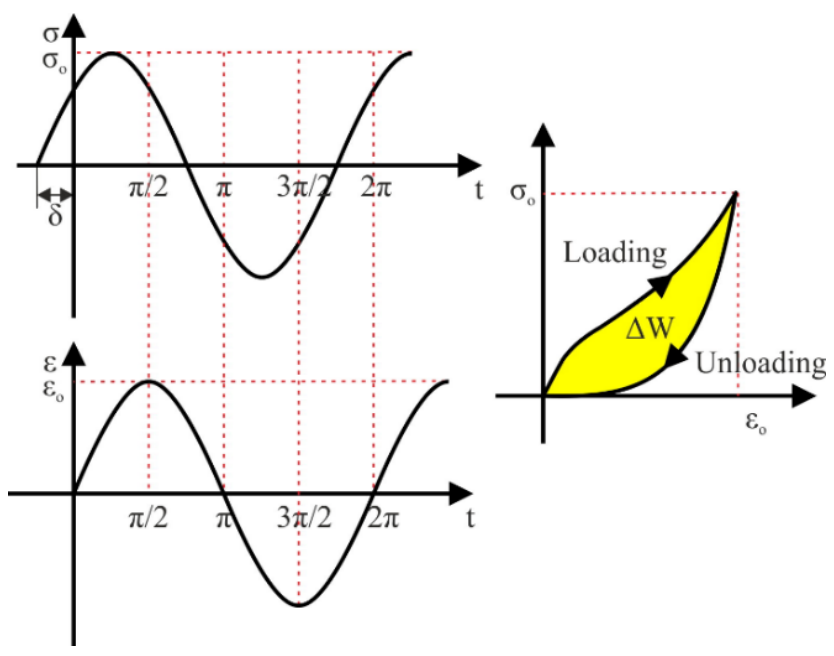

Fig. 1. Stress-strain hysteresis loops under fully reversed strain-controlled tension-compression.

The stress-strain curves of conventional NBR and NBR/MWCNTs mounts under uniaxial compression, as shown in Figure 2. For the same stress, it was found that the corresponding strain of the nanocomposite mount is lower than that of conventional NBR. Evidently, the stiffness of the nanocomposite mount was also improved by the addition of the MWCNTs, as shown in Table 1. This indicates that the NBR/MWCNTs composite system exploits the 
properties of one component to overcome the weakness of the other in mechanical performance.

Figure 2 presents the hysteresis loops curves of NBR/MWCNTs specimens under compressive vibration at $0.01 \mathrm{~Hz}$ with an ultimate force of $10 \mathrm{kN}$. Considering Eq. 2, the energy loss over a cycle $(\Delta W)$, the maximum energy of that cycle $(W)$ and loss factor $(n)$ were used to measure the material damping of the loading-unloading tests. $\Delta W$ indicates that the anti-vibration property of conventional NBR mounts is improved as the amount of MWCNTs increases. Therefore, the material is assumed to enhance the ability to transform its kinetics to those of thermal dissipation upon the application of an external load. Nevertheless, higher damping constant $n$, which is the ratio of $\Delta W$ to $W$, is observed for the NBR/MWCNTs mount and this indicates faster energy dissipation at particular amplitudes, which become stable with less vibration.

Table 1. The stiffness and damping values of NBR and NBR/MWCNTs mounts.

\begin{tabular}{|c|c|c|}
\hline Specimen & $\begin{array}{c}\text { Stiffness } \\
(\mathrm{N} / \mathrm{mm})\end{array}$ & Loss factor $n$ \\
\hline Conventional NBR mounts & 585 & $8.9 \%$ \\
\hline $20 \mathrm{wt} \% \mathrm{MWCNTs} / \mathrm{NBR}$ & 935 & $18.2 \%$ \\
\hline
\end{tabular}

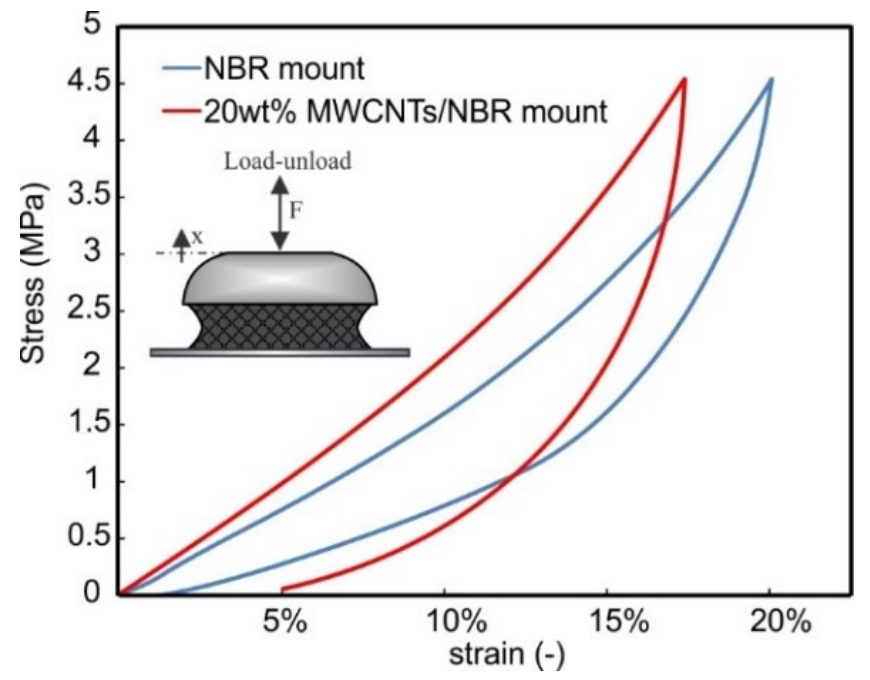

Fig. 2. Loading-unloading stress-strain curves of the mounts for different content of MWCNTs.

\subsection{Assessment of the vibration isolation performance of the elevator motor machine using finite element analysis (FEA)}

An elevator motor machine was used to apply the under study nanocomposite mounts. Firstly, a finite element model of the machine-isolator-ground system was introduced in order to assess the modal parameters and the vibration isolation performance of the existing mounts and the proposed nanocomposite ones. The computational model implemented in order to study the dynamic response of the elevator motor machine was achieved with the use of the finite element software code ANSYS APDL. Both a modal and a harmonic mode superposition analysis were performed. The total mass of the elevator motor machine was 
$106.8 \mathrm{~kg}$, which was used as gravitational static load. In order to determine the natural frequencies and participation factors, the modal analysis is carried out using the block Lanczos method with ANSYS for fixed-free boundary conditions. The modal superposition method enables one to express the response of a multi-degree-of-freedom system as a linear combination of its corresponding modal responses; to use free vibrations mode shapes to uncouple equations of motion. For the harmonic analysis, the motor load was applied and the response was acquired under the mounts at points 1, 2, 3 and 4, as shown in Figure 3(a).

The results of the transmissibility curves as estimated by the FEM are shown in Figure 3 (b). The vibration isolation behavior of the nanocomposite $20 \mathrm{wt} \% \mathrm{MWCNTs} / \mathrm{NBR}$ mount presented moderately higher resonant frequencies at $25 \mathrm{~Hz}, 134 \mathrm{~Hz}$ and $260 \mathrm{~Hz}$, since MWCNTs increase the stiffness of the elastomer in the isolator. More importantly, the transmissibility value at resonance is significantly decreased to $90 \%$. Thus, the $20 \mathrm{wt} \%$ MWCNTs/NBR mounts are considered as the optimal solution to reduce the vibration level of this elevator motor machine.
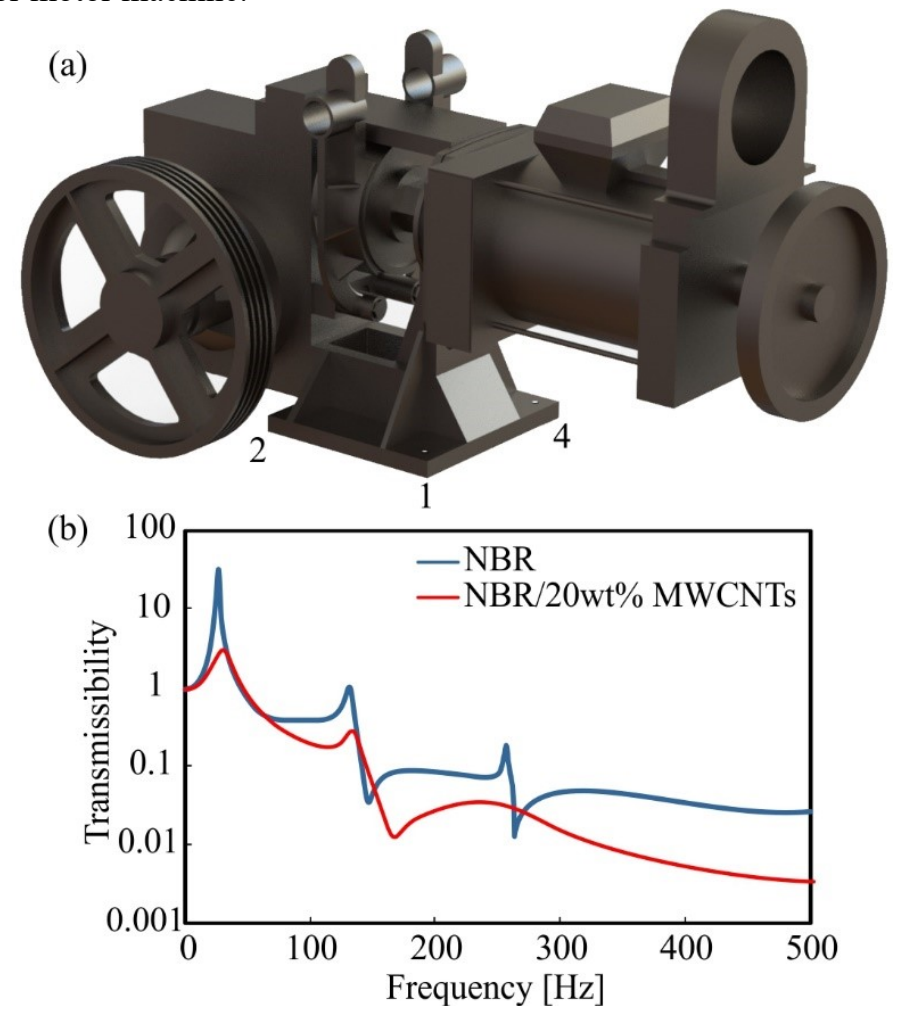

Fig. 3. (a) Characteristic measurement points for the dynamic analysis of the elevator motor machine and (b) evaluation of the transmissibility curve of the NBR and the nanocomposite mounts $20 \mathrm{wt} \%$ MWCNTs/NBR using FEA.

\section{Conclusions}

The overall objective of this paper is the estimation of the vibration isolation performance of an elevator engine machine mounted on conventional NBR isolators reinforced with MWCNTs. Cyclic compression test results showed higher stiffness and damping constant for the nanocomposite mount, which indicated faster energy dissipation at particular amplitudes, so they become stable with less vibration. The vibration isolation properties of the elevator motor machine were assessed by the transmissibility curves. The FEA results indicated that 
the addition of MWCNTs in the rubber-based composites significantly improved the vibration isolation performance of the machine compared with NBR mounts. Overall, the resonant frequencies, the damping and the capacity to isolate vibrations can be adjusted by modifying the content of MWCNTs in NBR mounts. The maximum percentage change of the transmissibility value at resonance reached a decrease of $90 \%$. Therefore, the $20 \mathrm{wt} \%$ MWCNTs/NBR mounts are considered effective vibration isolators for this elevator motor machine.

\section{References}

1. S. Neumark, Aer. Res. Coun. rep. and mem. no. 3269; (1962)

2. S.O. Oyadiji, and G.R. Tomlinson, J. Sound Vib.186 (4), 623-647 (1995)

3. U.J. Kurze, Act Acustica 2, 483-490 (1994)

4. R. Adams, J de Phys. Col; 44 (C9), C9-29-C9-37 (1983).

5. JB Kosmatka, SL Liguore, J Aerosp Eng 6:268-283 (1993)

6. A Baz, J. Ro, J Sound Vib 28, 18-21 (1994)

7. WH Liao, KW Wang, J Sound Vib 207:319-334 (1997)

8. M. Mansour, K. Tsongas, D. Tzetzis, A. Antoniadis, Polym Plast Technol Eng, 57, 171525 (2018)

9. M. Mansour, K. Tsongas, D. Tzetzis, Polym Plast Technol Eng, 58, 1234-44 (2019)

10. M. T. Mansour, K. Tsongas, D. Tzetzis, A. Antoniadis, IOP Conf. Ser.: Mater. Sci. Eng., 564 (2019)

11. D. Tzetzis, K. Tsongas, G. Mansour, Mater Res, 20(6), 1571-1578 (2017)

12. G. Mansour, K. Tsongas, D. Tzetzis, ICCM20, 20th Intern.l Conf. on Comp. Mat., 1924 July 2015, Copenhagen, Denmark.

13. K. Tsongas, D. Tzetzis, G. Mansour, Plast Rubber Compos, 46 (10) 458-468, (2017)

14. K. Tsongas, G. Mansour, Trib in Ind, 41 (3), 433-442 (2019)

15. G. Mansour, K. Tsongas, D. Tzetzis, Measurement 91, 31-38 (2016)

16. G. Mansour, K. Tsongas, D. Tzetzis, Compos Part B-Eng 94, 152-159 (2016)

17. G. Mansour, K. Tsongas, D. Tzetzis, J Reinf Plast Comp 35(19), 1401-1410 (2016)

18. G. Mansour, K. Tsongas, D. Tzetzis, K. Tzikas, , Polym Plast Technol Eng, 56(14), 1505-1515 (2017)

19. Sperling LH. Introduction to physical polymer science. 2nd ed. New York (NY): Wiley; 1992.

20. Rao SS. Mechanical vibrations. 5th ed. New Jersey (NJ): Prentice Hall; 2011.

21. Wevers M. NDT\&E Int. 1997;30(2):99-106.

22. G. Mansour, K. Tsongas, D. Tzetzis, J Balk Tribol Assoc, 21, 575-584, (2015) 